\title{
A novel risk score model based on five angiogenesis- related long non-coding RNAs for bladder urothelial carcinoma
}

\author{
Xinyuan Li ( $\sim$ lixinyuan@sibcb.ac.cn ) \\ Chongqing Medical University https://orcid.org/0000-0001-8385-8691 \\ Chunlin Zhang \\ Chongqing Medical University \\ Xiang Peng \\ Chongqing Medical University \\ Yang Li \\ Chongqing Medical University \\ Guo Chen \\ Chongqing Medical University \\ Xin Gou \\ Chongqing Medical University \\ Xiang Zhou \\ Chongqing Medical University \\ Chao Ma \\ Chongqing Medical University
}

\section{Research Article}

Keywords: bladder urothelial carcinoma, angiogenesis, tumour microenvironment, long noncoding RNA, risk model, prognosis.

Posted Date: February 25th, 2022

DOI: https://doi.org/10.21203/rs.3.rs-1198274/v1

License: (c) (i) This work is licensed under a Creative Commons Attribution 4.0 International License. Read Full License

Version of Record: A version of this preprint was published at Cancer Cell International on April 19th, 2022. See the published version at https://doi.org/10.1186/s12935-022-02575-1. 


\section{Abstract \\ Background}

Tumour angiogenesis is an independent risk factor for bladder urothelial carcinoma (BUC) progression, but viable and promising antiangiogenic targets are understudied. Long non-coding RNAs (IncRNAs) have been reported to play significant roles in angiogenesis.

\section{Methods}

The clinical data of BUC patients were obtained from TCGA database and clinical specimens of 138 BUC patients. Angiogenesis-related long non-coding RNAs (ARLNRs) were extracted from The Molecular Signatures Database v4.0. Univariate and multivariate COX regression analyses were used to further identify survival-related ARLNRs (sARLNRs). A chain of techniques (FACS, western blotting, qPCR, immunohistochemistry, tube formation, migration and invasion assays) and co-culture models were utilized to validate the clinical significances and angiogenetic correlation of SARLNRs.

\section{Results}

Five SARLNRs were employed to establish angiogenesis-related risk score model, by which the patients in the lowrisk group obtained longer overall survival than those in the high-risk group. Two sARLNRa in the risk score model (AC005625.1 and AC008760.1) expressed lower in BUC cell lines and tumour tissues than that in normal urothelial cells and adjacent normal tissues, with further lower expression in more advanced T stages. More importantly, the expression of AC005625.1 and AC008760.1 expression was negatively related to endothelial cells (ECs) percentage, tumour size and muscle invasion status. A prominently higher proportion of ECs was detected in tumour tissues with lower expression of AC005625.1 and AC008760.1. Furthermore, in the co-culture model of T24 cells and HUVEC cells, knockdown of AC005625.1 and AC008760.1 in BUC cells increased tube formation, migration and invasion abilities of HUVEC cells. The expression levels of CD31, VEGF-A, VIMENTIN and N-CADHERIN were also enhanced in HUVEC cells co-cultured with siR-AC005625.1 and siR-AC008760.1-treated T24 cells.

\section{Conclusion}

In the study, we identify five sARLNRs and validate their clinical significances, angiogenesis correlation and prognosis-predictive values in BUC. These results may provide a new perspective and some promising antiangiogenic targets for clinical diagnosis and treatment strategies of BUC.

\section{Background}

Bladder urothelial carcinoma (BUC) is the most common genitourinary malignancy with 500,000 new cases and 200,000 deaths per year worldwide ${ }^{1}$. BUC can present as non-muscle-invasive bladder cancer (NMIBC) accounting for near $80 \%$ patients diagnosed with BUC, muscle-invasive bladder cancer (MIBC) with higher aggression or as a metastatic disease ${ }^{2}$. Despite the 5 -year OS of NMIBC is greater than $85 \%$, it has not significantly improved for a good while, and the frequent recurrence also put a heavy load on the quality of life $(\mathrm{QOL})^{2-4}$. Representing the remaining 
$20 \%$ of localized disease, MIBC get the higher disease-specific mortality and the worse life expectancy, with approximately $50 \%$-year OS $^{1}$.

Although surgery, chemotherapy and immunotherapy have been widely applied for BUC, few survival improvements have been achieved until an emerging body of next-generation sequencing help us better understand the genetic variations and molecular alterations, identify actionable therapeutic targets, and predict patient prognosis ${ }^{5,6}$. Besides, the clear cellular geography and the acknowledge of various cell types allow us improve the awareness of genetic background better understand a considerable variety and heterogeneity of the tumour ${ }^{7}$. Recently, intense efforts are being made to clarify the genome-wide association of BUC and identify more valuable candidate genes for diagnostic markers, and therapeutic targets ${ }^{5}$. The variants of aldehyde dehydrogenase (ALDH2) enzyme were demonstrated to be closely associated with the higher recurrence rate of BUC ${ }^{8}$. CD44 polymorphisms probably responding for the susceptibility of BUC were potential for a molecular prognostic marker ${ }^{9}$. Additionally, the identification of some mutated genes such as FGFR3 ${ }^{10}$, TP53 ${ }^{11}$ and $\mathrm{ERCC} 2^{12,13}$, led to a resurgent interest in the realm of targeted therapy. An emerging body of studies at the genetic level are discovering more attractive and viable targets.

Tumour angiogenesis, a highly dynamic and complex pathological process of new vessel formation in the primary site or metastatic site, has been identified as an independent prognostic indicator in many cancers ${ }^{14,15}$. By supplying abundant nutrition and natural migration pathway, angiogenesis promotes tumour progression and regulates tumour microenvironment. Therefore, antiangiogenetic targeted therapy combined with anti-tumour cells approaches seems to be an alternative way for cancer therapy ${ }^{16}$. Additionally, identifying some promising angiogenetic markers has also been shown as a viable strategy for diagnosis and prognosis estimation, but is still in its infancy, especially for $\mathrm{BUC}^{17,18}$.

Long non-coding RNAs (IncRNAs), a group of non-coding RNAs that are more than 200 base pairs in length, are dynamically expressed in variety of biological activities including tumour angiogenesis ${ }^{19,20}$. LncRNAs affect angiogenesis by regulating various angiogenetic molecules, endothelial cell behaviors and tube formation. Besides, a cascade of IncRNAs have been found to participate in the angiogenesis-related pathways, such as vascular endothelial growth factor (VEGF) 21,22 and Notch ${ }^{23}$. Therefore, angiogenesis-related IncRNAs (ARIncR) as a kind of potential candidate, merit further discovery and attention in the field of antiangiogenetic strategies.

\section{Materials And Methods}

\section{Clinical sample collection}

Tumour tissues and adjacent normal tissues of 138 BUC patients who underwent tumour excision or tissue biopsy in the First Affiliated Hospital of Chongqing Medical University between March 2019 and February 2021 were collected. (Table 1) Patients with severe underlying diseases or other primary cancers were excluded. The collected clinical tissues were utilized to analyze the expression of IncRNAs and percentage of endothelial cells (ECs). All patients included in this study provided written informed consent, and this study was approved by the Medical Ethics Committee of the First Affiliated Hospital of Chongqing Medical University (IRB:2021-085). All clinical data were reviewed according to medical records.

\section{Cell Culture}


SV-HUC-1, BUC cell lines (T24, UM-UC-3, 5637, J82 and TCC-SUP) and HUVEC were purchased from the American Type Culture Collection (Manassas, Virginia, USA). Cells were cultured in DMEM (SV-HUC-1, UM-UC-3, T24 and HUVEC), McCoy's 5A (J82) and RPMI-1640 (5637 and TCC-SUP) basal medium (Gibco, Gaithersburg, MD, USA), which were supplemented with 10\% fetal bovine serum (FBS, MilliporeSigma, Burlington, MA, USA), 100 U/ml penicillin and $0.1 \mathrm{mg} / \mathrm{ml}$ streptomycin (Beyotime, Beijing, China). Cells were incubated at $37^{\circ} \mathrm{C}$ in $5 \% \mathrm{CO}_{2}$ incubator. The medium was changed every 1-3 days.

\section{Tissue processing}

Bladder specimens were obtained fresh from the operating field where grossly apparent tumour tissue or adjacent tissue not grossly affected by tumour (Par-cancer tissue). These tissues were transported at room temperature immersed in the RPMI-1640 medium (Gibco, Gaithersburg, MD, USA) with 10\% FBS (MilliporeSigma, Burlington, MA, USA). Once received, tumour tissues were divided into two parts, one of which was cut into approximately $1 \mathrm{~mm}^{3}$ pieces and enzymatically digested to single cell suspensions using MACS tumor dissociation kit (Miltenyi Biotec) for $1 \mathrm{~h}$ on a rotor at $37^{\circ} \mathrm{C}$ for further flow cytometry/FACS analysis, another part of tumour tissues and all par-cancer tissues were frozen in liquid nitrogen immediately and then stored at $-80^{\circ} \mathrm{C}$ until IncRNA extraction.

\section{ECs percentage detection by Flow cytometry/FACS}

The single cell suspensions were filtered with screen cloth and cell surface staining was performed in FACS buffer containing CD31 antibody (Cat\# 303102, BioLegend, USA) on ice for 1 hour. Following washing twice with FACS buffer, the percentages of EC subtype (CD31 positive) in these single cell suspensions were detected using a FACS flow cytometry system (Cytoflex, Beckman Coulter, USA).

\section{Cell transfection}

The siRNAs of AC005625.1 and AC008760.1 were used to silence the expression of AC005625.1 and AC008760.1. The sequences used were: si-AC005625.1 (sense:5'-GCUUCACAGCCACCAUCUATT-3', antisense: 5'-

UAGAUGGUGGCUGUGAAGCT T-3'), si-AC008760.1 (sense: 5'-GACAGGUAGUCACGACUAUTT-3', antisense: 5'-AUAGUC GUGACUACCUGUCTT-3'). For transient transfection, T24 cells were added into 6-well plate (1×10囚 cells per well). When cells grew to 50-60\% confluence, cells were transfected with $10 \mu$ l siRNAs (20 nM) using $5 \mu$ Lipofectamine 3000 (Invitrogen, USA) for $48 \mathrm{~h}$ according to the manufacturer. Finally, we used RT-qPCR to evaluate the expression levels of IncRNAs.

\section{Real-time quantitative PCR}

We used Trizol (Takara) to extract total IncRNAs from tissues and cell lines under various experimental conditions. cDNA Synthesis Kit (Takara) combined with IncRNAs $(1 \mu \mathrm{g})$ was utilized to reverse transcribed cDNA. The quantitative polymerase chain reaction (qPCR) was performed on an ABI 7500 real-time PCR system (Applied Biosystems) by SYBR-Green method (Takara). The values of Ct were calculated with the $2^{-\Delta \Delta C t}$ method and normalized to the expression levels of $\beta$-actin. The expression levels of IncRNAs were relative to the fold change of their controls which were defined as 1 . The primer sequences were shown in Table 2. Three assays were conducted per cDNA sample.

\section{Tube formation assay}


$100 \mu \mathrm{L}$ ice-cold Matrigel was added into a well of a 48-wells plate for the HUVEC cells tube formation assay. 1 hour

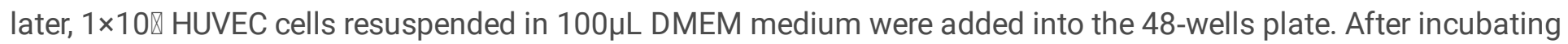
at $37^{\circ} \mathrm{C}$ in $5 \% \mathrm{CO}_{2}$ incubator for 4 hours, the tube formation status was photographed by light microscopy. The numbers of branch points were counted and analyzed by image J. 5 fields (200X) per chamber were observed for counting invaded cell numbers.

\section{Transwell assay}

For the migration assay, $1 \times 10 \otimes$ HUVEC cells were suspended in $100 \mu l$ medium without FBS and seeded into an upper chamber (Corning, USA). Then, $800 \mu \mathrm{l}$ complete DMEM medium containing $10 \%$ FBS was added to the lower chamber. For the invasion assay, Diluted Matrigel (1:5 dilution with the DMEM medium) was added into the cell culture inserts. Four hours later, $1 \times 10 \otimes$ HUVEC cells in $100 \mu$ non-FBS DMEM medium were added into the upper chamber, while the lower chamber was filled with $800 \mu$ of DMEM medium with $10 \%$ FBS. After culturing 24 hours for migration test and 48 hours for invasion test, the cells culture inserts were washed by PBS and stained with $4 \%$ paraformaldehyde. Then inserts were dyed by $0.1 \%$ crystal violet solution for 20 minutes. The dyed cells were photographed by light microscopy. 5 fields (200X) per chamber were observed for counting invaded cell numbers.

\section{Western blotting}

Total protein was extracted from HUVEC cells using Radio Immunoprecipitation Assay (RIPA) lysis buffer (Beyotime, Beijing, China) and 1\% PMSF (Beyotime). Next, the protein concentration was determined by the BCA Kit (Beyotime). The protein was separated by $10 \%$ sodium dodecyl sulfate polyacrylamide gel electrophoresis gel and transferred onto $0.22 \mu \mathrm{m}$ polyvinylidene fluoride (PVDF) membranes (Merck Millipore). The membranes were blocked with $5 \%$ milk in TBST buffer, and then the membranes were incubated with primary antibodies against VEGFA (Cat\# 65373, Cell Signaling Technology (CST), USA), CD31(Cat\# ab9498, Abcam, UK), VIMENTIN (Cat\# 5741, CST, USA), ECADHERIN (Cat\# 3195, CST, USA) and N-CADHERIN (Cat\# 13116, CST, USA) and $\beta$-Actin (Cat\# 4970, CST, USA) at $4^{\circ} \mathrm{C}$ for 16 hours. After washing with TBST buffer for 30 minutes, the membranes were incubated with anti-rabbit horseradish peroxidase-conjugate secondary antibodies (Cat\# 7074, CST, USA) at room temperature for 1 hour, and then washed by TBST 30 minutes. Signals were visualized by ECL Substrates (Beyotime). Three assays were conducted per protein sample. Protein bands were quantified by ImageJ software (U.S. National Institutes of Health). Target protein expression levels (band intensities) were expressed by relative to $\beta$-actin expression.

\section{Immunohistochemistry assay}

Immunohistochemistry (IHC) analysis was performed as described previously ${ }^{24}$. IHC was performed using antibodies against CD31 (Cat\# ab9498, Abcam, UK). Images were scanned using Pannoramic SCAN.

\section{BUC transcriptome data downloading and preprocessing}

Transcriptome RNA-sequencing data of 414 BUC tumour tissues and 19 normal tissues were downloaded and extracted from The Cancer Genome Atlas (TCGA) data portal (https://portal.gdc.cancer.gov/). We excluded patients whose OS $\leq 30$ days from this study because they might die of unpredictable factors such as hemorrhage and infection. The data utilized in the study were updated in March 21, 2021. Raw data of BUC patients were collected for further analyses. Transcriptome RNA-sequencing results and clinical data of BUC patients were combined into a matrix file by a merge script in the Perl language (http://www.perl.org/). 
Angiogenesis-related genes (ARGs) were extracted from The Molecular Signatures Database v4.0 (ANGIOGENESIS M14493 and WP_ANGIOGENESIS M39556, http://www. broadinstitute.org/gsea/msigdb/index.jsp). Then the angiogenetic scores of these ARGs were calculated according to their expression levels in BUC tissues. To further identify the ARLNRs, we conducted the Pearson correlation analysis to clarify the correlation between angiogenetic score and the expression of IncRNA in BUC tissue. A standard of $|r|>0.6$ and $P<0.05$ was used to screen the ARLNRs. Besides, we selected the sARLNRs by univariate COX analysis and survival packages of R software $(P<0.01)$. SARLNRs were further divided into deleterious and protective portions by the Hazard ratio (HR).

\section{Establish angiogenesis-related risk score model (ARRSM)}

Through multivariate COX regression analysis, we established the ARRSM based on the selected sARLNRs. The score in the ARRSM was calculated based on the expression of SARLNRs together with the Cox regression coefficients. The formula was as followed, [Expression levels of KIRREL1-IT1 * $(0.266525)$ ] + [Expression levels of AC005625.1 * (-0.135165)] + [Expression levels of AC018809.1 * (-0.170812)] + [Expression levels of AC008760.1 * $(-0.133273)]+[$ Expression levels of AC083862.2 * $(-0.221852)]$. BUC patients were separated into the high-risk group and the low-risk group according to the median score.

\section{Bioinformatics analysis}

Receiver operating characteristic (ROC) curves were used to assess the sensitivity and specificity of the ARRSM and drawn by survival ROC package of R software. Gene set enrichment analysis (GSEA) was used to detect the different pathways of ARRSM. We evaluated the survival probabilities of patients in different risk groups by Kaplan-Meier survival curves. We conducted the univariate and multivariate Cox regression analyses to verify the independent prognostic factor of BUC. Nomograms were drawn to predict the survival probabilities of BUC patients by the rms package of $\mathrm{R}$ software.

\section{Statistical analysis}

Statistical analysis was conducted by SPSS21.0 software (SPSS Inc, Chicago, IL) and GraphPad Prism8 (GraphPad Software Inc, La Jolla, CA). Data were expressed as means \pm SD. The correlations between average expression of SARLNRs and clinicopathological characteristics of patients were evaluated using Fisher's exact probability method. Student T-test, ANOVA and post-hoc test (Boferroni method) were used for difference comparison of two or more groups. Pearson correlation analysis was utilized to analyze the correlation. $P<0.05$ was considered a significantly statistical difference.

\section{Results}

\section{Acquisition of SARLNRs}

Transcriptome RNA-sequencing data and clinical data of BUC patients were downloaded from TCGA database. Following that, IncRNAs data were extracted from the transcriptome data and 1282 differentially expressed IncRNAs were identified by limma algorithm, of which 310 IncRNAs were down-regulated and 972 IncRNAs were upregulated (Fig. 1A and 1B). Then, we screened 72 ARGs in the ANGIOGENESIS M14493 and WP_ANGIOGENESIS M39556 of Molecular Signatures Database. After analyzing the correlations of the 1282 differentially expressed IncRNAs and 72 ARGs by Pearson correlation analysis, we found that 98 IncRNAs were associated with

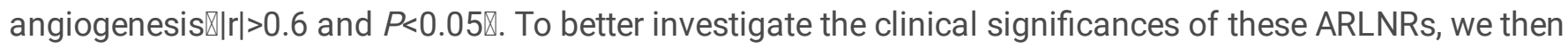
conducted the univariate COX regression analysis. Finally, 10 ARLNRs were identified to be relevant to OS of BUC 
patients (sARLNRs: AL157392.3, AP000346.1, KIRREL1-IT1, AP002840.2, AC005625.1, AL356512.1, AC018809.1, IGBP1-AS1, AC008760.1 and AC083862.2) ( $P<0.05)$. As shown in the forest map, all sARLNRs but aside from KIRREL1-IT1 were regarded as protective factors (Fig. 1C).

\section{Establish the angiogenesis-related risk score model}

To further validate the survival and prognostic relevance, we conducted multivariate COX regression analysis. Finally, KIRREL1-IT1, AC005625.1, AC018809.1, AC008760.1 and AC083862.2 were employed to establish the ARRSM (Table $3)$. Besides, the correlations of the five sARLNRs and OS were further illustrated via the survival curves in which the higher expression levels of AC005625.1, AC018809.1, AC008760.1 and AC083862.2 were correlated with the longer survival time, while the expression levels of KIRREL1-IT1 showed the opposite conclusion (Fig. S1). According to the median risk score, BUC patients were divided into the high-risk group and the low-risk group (Fig. 1D). With the increasing risk score, the mortality rate of BUC patients and the expression levels of KIRREL1-IT1 constantly increased, while AC005625.1, AC018809.1, AC008760.1 and AC083862.2 expressed decreasingly (Fig. 1E). KaplanMeier analysis illuminated that BUC patients in the low-risk group exhibited a longer survival time than those in the high-risk group (Fig. 1F).

\section{The relationship of ARRSM and clinicopathological feature}

In order to explore the relevance of SARLNRs and clinical features of BUC patients, we used ggpubr package of R language to analyze the correlations of ARRSM and the clinical characteristics, such as grade, stage, TNM stage. We found the expression levels of AC018809.1 and AC008760.1 were enhanced in patients with advanced grade (Fig. 2A). The expression levels of AC005625.1 and AC008760.1 increased in the more advanced stage while KIRREL1-IT1 dropped (Fig. 2B). Besides, the expression level of KIRREL1-IT1 was elevated in the advanced T-stage but AC008760.1 decreased (Fig. 2C). AC005625.1 expressed more in the advanced N-stage and M-stage (Fig. 2D and 2E).

\section{The clinical application of ARRSM and Gene Set Enrichment Analysis (GESA)}

Next, we performed univariate analysis to analyze the independent risk factor of BUC patients, and the results showed gender, stage, T-stage, $\mathrm{N}$-stage, $\mathrm{M}$-stage and risk score were significantly correlated to prognosis $(P<0.05)$. However, through the multivariate analysis, only risk score could be served as an independent risk factor of BUC patients (Table 4). Next, we calculated the areas under curves (AUCs) for receiver operating characteristic (ROC) curves of ARRSM and clinical characters, and found the AUCs of risk score, age, gender, grade, stage, T-stage, Mstage and $\mathrm{N}$-stage were $0.741,0.57,0.486,0.422,0.659,0.651,0.521$ and 0.629 respectively (Fig. 2F). The highest AUC was risk score, which meant the ARRSM was the most accuracy independent risk factor of BUC patients. We normalized the points of each BUC patient to a distribution ranging from 0 to 100 , and then we calculated the 1-year, 3-year and 5-year survival probabilities of BUC patients by drawing a vertical line (clinical features and the expression levels of sARLNRs) between the total points axis and each prognosis axis (Fig. 3A and 3B). The nomograms will provide a novel diagnostic method for clinical workers to assess the prognosis of BUC patients. These results illustrated that the ARRSM could be served as a promising risk score model in predicting the prognosis of BUC patients.

Then, we used kyoto encyclopedia of genes and genomes (KEGG) pathway analysis of GSEA to further investigate the underlying mechanisms of ARRSM. We found that the high-risk group was closely related to the VEGF signaling 
pathway (Fig. 3C). These results motivated us to further discover the underlying mechanisms of angiogenesis and immune cell infiltration in the future studies.

\section{AC005625.1 and AC008760.1 expressed lowly in BUC cell lines and tumour tissues, especially in patients with more advanced T stages}

In order to verify the expression levels of sARLNRs in various BUC cell lines and clinical samples, we detected the expression levels of AC005625.1 and AC008760.1 in SV-HUC-1, different BUC cell lines, tumour tissues and adjacent normal tissues. Compared with SV-HUC-1, the expression levels of AC005625.1 and AC008760.1 were significantly lower in T24, 5637, UMUC-3, J82 and TCC-SUP BUC cell lines (Fig. 4A). Besides, we found that AC005625.1 and AC008760.1 expressed lower in tumour tissues than those in adjacent normal tissues (Fig. 4B), with the lower expression in more advanced T stage (Fig. 4C).

\section{AC005625.1 and AC008760.1 expression was negatively related to ECs percentage, tumour size and muscle invasion status}

To further investigate the clinical significance of the sARLNRs, we first divided the 138 BUC patients into sARLNRshigh-expression ( $n=69)$ and sARLNRs-low-expression $(n=69)$ groups according to the average expression of AC005625.1 and AC008760.1 (the cut-off value: 0.4057). As illustrated in Table 1, the expression of AC005625.1 and AC008760.1 was significantly related to ECs percentage, tumour size and muscle invasion status, while there was no remarkable correlation with gender, age, distant metastasis, lymph node status, or nidus number. To further discover the correlation of the SARLNRs and tumour-related angiogenesis, we detected the percentage of EC subtype in tumour tissues by FACS. As illustrated in Fig. 4D and 4E, a prominently higher proportion of EC was observed in tumours with lower average expression of AC005625.1 and AC008760.1. The result of Pearson correlation analysis suggested the significant negative correlation between SARLNRs expression and ECs percentage $(r=-0.599, P]$ 0.001) (Fig. 4F). Furthermore, the higher percentage of EC in tumour tissues with lower expression of AC005625.1 and AC008760.1 was also validated by IHC (Fig. 4G).

\section{Knockdown of AC005625.1 and AC008760.1 in BUC cells promoted tube formation, migration and invasion of HUVECS}

To further determine the effects of AC005625.1 and AC008760.1 in tumour-related angiogenesis, we knocked down the AC005625.1 and AC008760.1 using siRNAs in T24 cells (Fig. 5A) followed by the co-culture of T24 cells and HUVEC cells to simulate tumour microenvironment of BUC (Fig. 5B). Next, we performed HUVEC cells tube formation and Transwell experiments to explore the angiogenesis-related roles of AC005625.1 and AC008760.1 in tumour microenvironment of BUC. As shown in Fig. 5C and 5D, the tube formation, migration and invasion abilities of HUVEC cells co-cultured with siRNAs-treated T24 cells were prominently enhanced. Then, we found that the expression of angiogenesis-related proteins (CD31 and VEGF-A) (Fig. 5E) and the key proteins in the epithelialmesenchymal transition pathway (VIMENTIN and N-CADHERIN) (Fig. 5F) increased in HUVECs which were cocultured with siR-AC005625.1 and siR-AC008760.1-treated T24 cells, while E-CADHERIN decreased. These results

demonstrated that knockdown of AC005625.1 and AC008760.1 in BUC cells promoted tumour-related angiogenesis by augmenting the tube formation, invasion and migration abilities of HUVECs in tumour microenvironment.

\section{Discussion}


Increasing evidence has unraveled that angiogenesis is crucial for tumour progression and is highly dependent on VEGF expressed by most malignant tumours ${ }^{25-27}$. Additionally, FRS2-mediated signals were validated to promote tumour angiogenesis and predict poor prognosis in prostate carcinoma, high-grade serous ovarian cancer and liposarcoma ${ }^{28-30}$. The finding of recurrent ADGRG6 enhancer mutations also facilitated our knowledge of underlying molecular mechanisms of pathological angiogenesis in the highly vascularized cancers ${ }^{31}$. Hypoxiainducible factor 1 (HIF1) as a heterodimeric transcription factor composed of HIF1a and HIF1 $\beta$ subunits, is also regarded as a major angiogenetic regulator in the tumour microenvironment ${ }^{32}$. Recently, a phase II trial illuminated that ramucirumab (a VEGF receptor-2 antibody) combined with second-line docetaxel, seemed to show the beneficial outcomes $^{33}$. However, anti-angiogenesis has not yet been verified as a therapy strategy with higher priority in urothelial carcinoma. Besides, much less is known about the potential roles of angiogenesis-related biomarkers.

Recent studies have demonstrated that IncRNAs regulated the various processes involved in angiogenesis directly or indirectly by targeting different angiogenetic molecules ${ }^{34}$. Because of the influential roles, IncRNAs are rapidly emerging as a type of promising drug target and candidate. Lin et al. reported that IncRNA UBE2CP3 augmented hepatocellular carcinoma cell secretion of VEGFA and promoted angiogenesis by activating ERK1/2/HIF1a/VEGFA axis $^{35}$. LncRNA PAXIP1-AS1 was identified to boost migration and angiogenesis of glioma by upregulating ETS1midiated KIF14 expression ${ }^{36}$. Aside from the pro-angiogenetic potential, Chang et al. found IncRNA LINC00320 suppressed tumourigenicity of glioma and angiogenesis through reduction of NFKB1-regulated AQP9 ${ }^{37}$. A growing body of ARIncRs not only provided therapeutic targets, but were also promising for diagnosis and prognosis evaluation. For example, IncRNA PANTR1 was related to poor prognosis and promoted angiogenesis and apoptosis in clear cell renal cell cancer ${ }^{38}$.

In this study, we identified five ARIncRs with prominent clinical significance of BUC. Following the establishment of ARRSM based on the five ARIncRs, BUC patients could be divided into the high-risk group and the low-risk group by scores reflecting the different OS. Besides, through the in vitro experiments, we not only found the lower expression of AC005625.1 and AC008760.1 in BUC cells and tumour tissues, but also validated the negative correlation with T stage, tumour size and muscle invasion status. More importantly, we found that AC005625.1 and AC008760.1 expression was negatively related to ECs percentage in tumour tissues. Besides, the angiogenesis-inhibited roles and underlying mechanisms of AC005625.1 and AC008760.1 were further validated in the co-culture models of HUVEC cells and T24 cells.

Identified as an autophagy-related IncRNA, AC008760.1 was demonstrated to be potential for predicting poor prognosis of colorectal cancer ${ }^{39}$, but few studies reported the roles of KIRREL1-IT1, AC005625.1, AC018809.1, AC008760.1 and AC083862.2 in the realm of angiogenesis. It is clear that tumour vascularization, as a hallmark feature of cancer, is not a just innocent bystander, but in many cases regulate crucial process of tumour progression. Therefore, identifying more promising angiogenetic targets merit further attention. Here, we established an ARRSM based on five SARLNRs, which not only provided more therapeutic targets, but also better evaluate prognosis of BUC patients from the perspective of tumour vascularization.

Although we elucidated the potential of ARRSM for prognosis assessment of BUC patients and validated clinical significances and angiogenetic correlation of sARLNRs through a series of in vitro experiments based on cell lines and clinical samples, some limitations remain to be further strengthened in future study. First, proteomics and metabonomics assays should be implemented to reveal wider and deeper perspectives on tumour angiogenesis. Next, the more underlying mechanisms of these SARLNRs of ARRSM should be explored through a chain of in vivo and in vitro experiments in subsequent study. 


\section{Conclusion}

In the present manuscript, we unraveled the potential of SARLNRs for prognosis evaluation of BUC from the angiogenetic perspective and ascertained their clinical significance. These findings not only help to establish a link between IncRNA and tumour angiogenesis, but also provide a reliable and accurate ARRSM developed based on some novel targets for future antiangiogenic therapies.

\section{Abbreviations}

ARLNRs

angiogenesis-related long non-coding RNAs

ARRSM

angiogenesis-related risk score model

BUC

Bladder urothelial carcinoma

FBS

Fetal bovine serumGSEA:gene set enrichment analysisIME:immune microenvironment

MIBC

muscle-invasive bladder cancer

NMIBC

non-muscle-invasive bladder cancer

OS

overall survival

QOL

quality of life

sARLNRs

survival-related ARLNRs

IncRNAs

Long non-coding RNAs

VEGF

vascular endothelial growth factor

\section{Declarations}

\section{Ethics approval and consent to participate}

Informed consent forms have been signed by all patients before this study. The research protocol has been approved by the Ethics Committee of The First Affiliated Hospital of Chongqing Medical University and is based on the ethical principles of medical research involving human subjects in the Helsinki Declaration.

\section{Consent for publication}

Not applicable.

\section{Availability of data and materials}

Authors can provide all of datasets analyzed during the study on reasonable request. 


\section{Competing interests}

The authors report no competing interests in this work.

\section{Funding}

None.

\section{Authors' contributions}

Xinyuan Li, Xin Gou, Xiang Zhou and Chao Ma designed the study. Xinyuan Li wrote the manuscript and analyzed data. Xiang Zhou performed the bioinformatics analysis. Chunlin Zhang and Xiang Peng collected the samples and verified the gene expression level. Yang Li and Guo Chen conducted the tube formation, migration and invasion assays.

\section{Acknowledgements}

Not applicable.

\section{References}

1. Dobruch, J. et al. Gender and Bladder Cancer: A Collaborative Review of Etiology, Biology, and Outcomes. 69, 300-310, doi:10.1016/j.eururo.2015.08.037 (2016).

2. Tran, L., Xiao, J., Agarwal, N., Duex, J. \& Theodorescu, D. J. N. r. C. Advances in bladder cancer biology and therapy. 21, 104-121, doi:10.1038/s41568-020-00313-1 (2021).

3. Smith, A. et al. Impact of bladder cancer on health-related quality of life. 121, 549-557, doi:10.1111/bju.14047 (2018).

4. Dizman, N., Meza, L. \& Pal, S. J. N. m. Biomarker approach harnessed in trials of personalized medicine for bladder cancer. 27, 761-763, doi:10.1038/s41591-021-01300-1 (2021).

5. Hurst, C. et al. Genomic Subtypes of Non-invasive Bladder Cancer with Distinct Metabolic Profile and Female Gender Bias in KDM6A Mutation Frequency. 32, 701-715.e707, doi:10.1016/j.ccell.2017.08.005 (2017).

6. Piao, X., Byun, Y., Kim, W., Kim, J. J. I. \& urology, c. Unmasking molecular profiles of bladder cancer. 59, 72-82, doi:10.4111/icu.2018.59.2.72 (2018).

7. Carrot-Zhang, J. et al. Comprehensive Analysis of Genetic Ancestry and Its Molecular Correlates in Cancer. 37, 639-654.e636, doi:10.1016/j.ccell.2020.04.012 (2020).

8. Xi, X., Chen, S., Mi, H. J. E. \& medicine, t. Aldh2 gene reduces oxidative stress in the bladder by regulating the NFKB pathway in a mouse model of ketamine-induced cystitis. 20, 111, doi:10.3892/etm.2020.9239 (2020).

9. Woodman, A. et al. Analysis of anomalous CD44 gene expression in human breast, bladder, and colon cancer and correlation of observed mRNA and protein isoforms. 149, 1519-1530 (1996).

10. Loeffler, C. et al. Artificial Intelligence-based Detection of FGFR3 Mutational Status Directly from Routine Histology in Bladder Cancer: A Possible Preselection for Molecular Testing? , doi:10.1016/j.euf.2021.04.007 (2021).

11. Manzano, R., Catalan-Latorre, A. \& Brugarolas, A. J. B. c. RB1 and TP53 co-mutations correlate strongly with genomic biomarkers of response to immunity checkpoint inhibitors in urothelial bladder cancer. 21, 432, doi:10.1186/s12885-021-08078-y (2021). 
12. Hirotsu, Y. et al. ERCC2Genomic Profiling Identified E606Q Mutation in Helicase Domain Respond to PlatinumBased Neoadjuvant Therapy in Urothelial Bladder Cancer. 10, 1643, doi:10.3389/fonc.2020.01643 (2020).

13. Bellmunt, J. et al. Genomic Predictors of Good Outcome, Recurrence, or Progression in High-Grade T1 NonMuscle-Invasive Bladder Cancer. 80, 4476-4486, doi:10.1158/0008-5472.Can-20-0977 (2020).

14. Huinen, Z., Huijbers, E., van Beijnum, J., Nowak-Sliwinska, P. \& Griffioen, A. J. N. r. C. o. Anti-angiogenic agents overcoming tumour endothelial cell anergy and improving immunotherapy outcomes. doi:10.1038/s41571-02100496-y (2021).

15. Bellmunt, J. J. L. Antiangiogenesis to curb urothelial cancer. 390, 2220-2221, doi:10.1016/s01406736(17)32388-7 (2017).

16. Vogelzang, N. J. J. o. c. o. o. j. o. t. A. S. o. C. O. Antiangiogenic agents, chemotherapy, and the treatment of metastatic transitional cell carcinoma. 31, 670-675, doi:10.1200/jco.2012.44.4349 (2013).

17. Gallagher, D. et al. Phase II study of sunitinib in patients with metastatic urothelial cancer. 28, 1373-1379, doi:10.1200/jco.2009.25.3922 (2010).

18. de Bono, J. et al. Tisotumab vedotin in patients with advanced or metastatic solid tumours (InnovaTV 201): a first-in-human, multicentre, phase 1-2 trial. 20, 383-393, doi:10.1016/s1470-2045(18)30859-3 (2019).

19. Su, M. et al. Long non-coding RNAs in esophageal cancer: molecular mechanisms, functions, and potential applications. 11, 118, doi:10.1186/s13045-018-0663-8 (2018).

20. Pichler, M. et al. Therapeutic potential of FLANC, a novel primate-specific long non-coding RNA in colorectal cancer. 69, 1818-1831, doi:10.1136/gutjnl-2019-318903 (2020).

21. Peng, Z., Liu, C. \& Wu, M. J. M. c. New insights into long noncoding RNAs and their roles in glioma. 17, 61, doi:10.1186/s12943-018-0812-2 (2018).

22. He, Y. et al. Long non-coding RNA GAPLINC promotes angiogenesis by regulating miR-211 under hypoxia in human umbilical vein endothelial cells. 23, 8090-8100, doi:10.1111/jcmm.14678 (2019).

23. Pan, Y., Mao, Y., Jin, R. \& Jiang, L. J. O. I. Crosstalk between the Notch signaling pathway and non-coding RNAs in gastrointestinal cancers. 15, 31-40, doi:10.3892/ol.2017.7294 (2018).

24. Li, X. et al. Secretory autophagy-induced bladder tumour-derived extracellular vesicle secretion promotes angiogenesis by activating the TPX2-mediated phosphorylation of the AURKA-PI3K-AKT axis. Cancer Lett 523, 10-28, doi:10.1016/j.canlet.2021.09.036 (2021).

25. Ash, D. et al. The P-type ATPase transporter ATP7A promotes angiogenesis by limiting autophagic degradation of VEGFR2. Nature communications 12, 3091, doi:10.1038/s41467-021-23408-1 (2021).

26. Kim, S. et al. Comprehensive molecular characterization of gastric cancer patients from phase II second-line ramucirumab plus paclitaxel therapy trial. Genome medicine 13, 11, doi:10.1186/s13073-021-00826-w (2021).

27. Shashni, B., Nishikawa, Y. \& Nagasaki, Y. Management of tumor growth and angiogenesis in triple-negative breast cancer by using redox nanoparticles. Biomaterials 269, 120645, doi:10.1016/j.biomaterials.2020.120645 (2021).

28. Luo, L. et al. The Tyrosine Kinase Adaptor Protein FRS2 Is Oncogenic and Amplified in High-Grade Serous Ovarian Cancer. Molecular cancer research : MCR 13, 502-509, doi:10.1158/1541-7786.Mcr-14-0407 (2015).

29. Hanes, R. et al. Preclinical Evaluation of the Pan-FGFR Inhibitor LY2874455 in FRS2-Amplified Liposarcoma. Cells 8, doi:10.3390/cells8020189 (2019).

30. Liu, J. et al. Hyperactivated FRS2a-mediated signaling in prostate cancer cells promotes tumor angiogenesis and predicts poor clinical outcome of patients. Oncogene 35, 1750-1759, doi:10.1038/onc.2015.239 (2016).

Page $12 / 21$ 
31. Wu, S. et al. Whole-genome sequencing identifies ADGRG6 enhancer mutations and FRS2 duplications as angiogenesis-related drivers in bladder cancer. Nature communications 10, 720, doi:10.1038/s41467-01908576-5 (2019).

32. de Heer, E., Jalving, M. \& Harris, A. HIFs, angiogenesis, and metabolism: elusive enemies in breast cancer. The Journal of clinical investigation 130, 5074-5087, doi:10.1172/jci137552 (2020).

33. Sonpavde, G. \& Bellmunt, J. Bladder cancer: Angiogenesis as a therapeutic target in urothelial carcinoma. Nature reviews. Urology 13, 306-307, doi:10.1038/nrurol.2016.69 (2016).

34. Kumar, M. \& Goyal, R. LncRNA as a Therapeutic Target for Angiogenesis. Current topics in medicinal chemistry 17, 1750-1757, doi:10.2174/1568026617666161116144744 (2017).

35. Lin, J. et al. Long non-coding RNA UBE2CP3 enhances HCC cell secretion of VEGFA and promotes angiogenesis by activating ERK1/2/HIF-1a/VEGFA signalling in hepatocellular carcinoma. Journal of experimental clinical cancer research : CR 37, 113, doi:10.1186/s13046-018-0727-1 (2018).

36. Xu, H. et al. Long non-coding RNA PAXIP1-AS1 facilitates cell invasion and angiogenesis of glioma by recruiting transcription factor ETS1 to upregulate KIF14 expression. Journal of experimental clinical cancer research : CR 38, 486, doi:10.1186/s13046-019-1474-7 (2019).

37. Chang, L. et al. Long Non-coding RNA LINC00320 Inhibits Tumorigenicity of Glioma Cells and Angiogenesis Through Downregulation of NFKB1-Mediated AQP9. Frontiers in cellular neuroscience 14, 542552, doi:10.3389/fncel.2020.542552 (2020).

38. Seles, M. et al. Long Non-Coding RNA PANTR1 is Associated with Poor Prognosis and Influences Angiogenesis and Apoptosis in Clear-Cell Renal Cell Cancer. Cancers 12, doi:10.3390/cancers12051200 (2020).

39. Wei, J. et al. An Autophagy-Related Long Noncoding RNA Signature Contributes to Poor Prognosis in Colorectal Cancer. Journal of oncology 2020, 4728947, doi:10.1155/2020/4728947 (2020).

\section{Tables}

Table 1: Relationship between AC005625.1 and AC008760.1expression and clinicopathologic factors of UBC patients 


\begin{tabular}{|c|c|c|c|c|}
\hline \multirow[t]{2}{*}{ Parameter } & \multirow[t]{2}{*}{$\mathbf{N}$} & \multicolumn{2}{|c|}{ Average Expression of sARLNRs } & \multirow[t]{2}{*}{$P$ value } \\
\hline & & Low & High & \\
\hline Gender & & & & 0.361 \\
\hline Male & 115 & 60 & 55 & \\
\hline Female & 23 & 9 & 14 & \\
\hline Age (year) & & & & 0.305 \\
\hline$\square 65$ & 75 & 34 & 41 & \\
\hline$\geq 65$ & 63 & 35 & 28 & \\
\hline ECs percentage $(\%)$ & & & & $<0.001$ \\
\hline$\square 25$ & 69 & 18 & 51 & \\
\hline$\geq 25$ & 69 & 51 & 18 & \\
\hline Tumor size (cm) & & & & 0.003 \\
\hline$\square 2$ & 58 & 20 & 38 & \\
\hline$\geq 2$ & 80 & 49 & 31 & \\
\hline Muscle invasion status & & & & 0.022 \\
\hline Negative & 52 & 19 & 33 & \\
\hline Positive & 86 & 50 & 36 & \\
\hline Lymph node status & & & & 0.208 \\
\hline Negative & 132 & 64 & 68 & \\
\hline Positive & 6 & 5 & 1 & \\
\hline Distant metastasis status & & & & 0.098 \\
\hline Negative & 123 & 58 & 65 & \\
\hline Positive & 15 & 11 & 4 & \\
\hline Nidus number & & & & \\
\hline Single & 126 & 64 & 62 & 0.764 \\
\hline Multiple & 12 & 5 & 7 & \\
\hline
\end{tabular}

Note: The bold number represents the $P$-values with significant differences.

Table 2: The primer sequences of AC005625.1, AC008760.1 and $\beta$-actin 


\begin{tabular}{|lll|}
\hline AC005625.1 & F primer (5'-3') & TTGTTTGTTGTTCGCCACC \\
\cline { 2 - 3 } & R primer (5'-3') & CGCTGCCCAATCCCTTCA \\
\hline AC008760.1 & F primer (5'-3') & TCCTGAGATGAAGCTGGAAATCAA \\
\cline { 2 - 3 } B-actin & R primer (5'-3') & AGTTTCTACGGTGGAGGGGT \\
& R primer (5'-3') & AAACGTGCTGCTGACCGAG \\
\hline
\end{tabular}

Note: F primer: forward primer; $\mathrm{R}$ primer: reverse primer.

Table 3: The results of multivariate Cox regression analysis

\begin{tabular}{|llllll|}
\hline Gene & Coefficients & HR & HR.95\% high & HR.95\% low & P value \\
\hline KIRREL1-IT1 & 0.26652 & 1.30542 & 1.58871 & 1.07264 & 0.00782 \\
\hline AC005625.1 & -0.13517 & 0.87357 & 1.03632 & 0.73638 & 0.1209 \\
\hline AC018809.1 & -0.17081 & 0.84297 & 0.96365 & 0.73741 & 0.01233 \\
\hline AC008760.1 & -0.13327 & 0.87522 & 0.99154 & 0.77254 & 0.03632 \\
\hline AC083862.2 & -0.22185 & 0.80103 & 0.93114 & 0.68911 & 0.00386 \\
\hline
\end{tabular}

Note: HR: Hazard Ratio.

Table 4: Univariate and multivariate COX analysis of UBC patients

\begin{tabular}{|lllllllll|}
\hline \multicolumn{4}{c}{ Univariate analysis } & \multicolumn{5}{l|}{ Multivariate analysis } \\
Variables & HR & $\begin{array}{l}\text { HR 95\% } \\
\text { low }\end{array}$ & $\begin{array}{l}\text { HR 95\% } \\
\text { high }\end{array}$ & P value & HR & $\begin{array}{l}\text { HR 95\% } \\
\text { low }\end{array}$ & $\begin{array}{l}\text { HR 95\% } \\
\text { high }\end{array}$ & P value \\
\hline Age & 1.023152 & 0.997282 & 1.049694 & 0.079829 & 1.012561 & 0.985234 & 1.040647 & 0.371155 \\
\hline Gender & 0.592245 & 0.353164 & 0.993178 & 0.047042 & 0.625978 & 0.364538 & 1.074915 & 0.089491 \\
\hline Stage & 1.804371 & 1.290509 & 2.522841 & 0.000557 & 1.187281 & 0.595591 & 2.366786 & 0.625752 \\
\hline T-stage & 1.684872 & 1.173265 & 2.419568 & 0.004722 & 1.359828 & 0.826785 & 2.236534 & 0.226006 \\
\hline M-stage & 2.520036 & 1.005311 & 6.317033 & 0.048694 & 1.456805 & 0.526254 & 4.032801 & 0.468921 \\
\hline N-stage & 1.556737 & 1.207657 & 2.006723 & 0.000634 & 1.280255 & 0.797118 & 2.056222 & 0.306788 \\
\hline $\begin{array}{l}\text { Risk } \\
\text { score }\end{array}$ & 2.043276 & 1.573784 & 2.652826 & $8.12 \mathrm{e}-08$ & 2.054913 & 1.558068 & 2.710195 & $3.39 \mathrm{e}-07$ \\
\hline
\end{tabular}


Note: HR: Hazard Ratio.

\section{Figures}

\section{Figure 1}

A

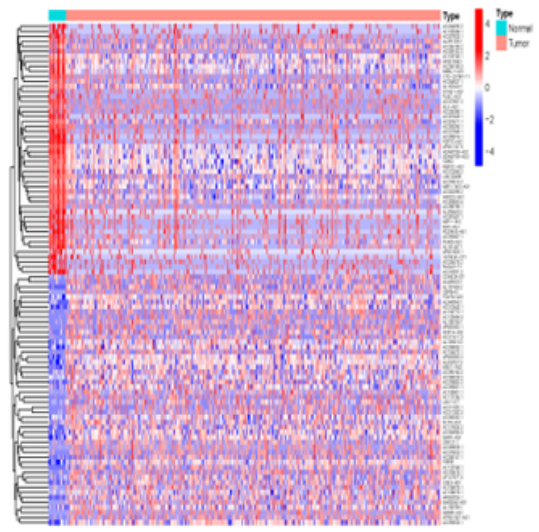

D

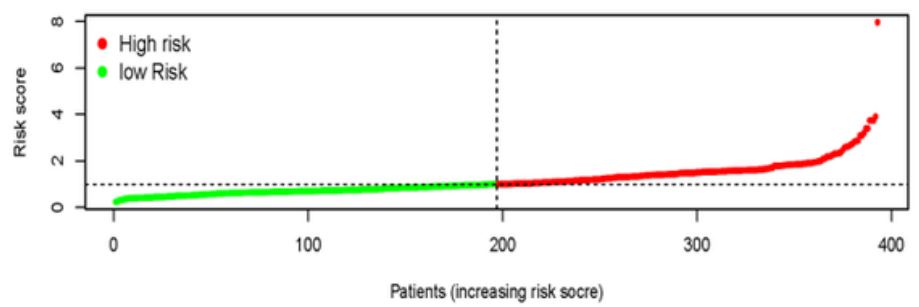

$\mathrm{E}$

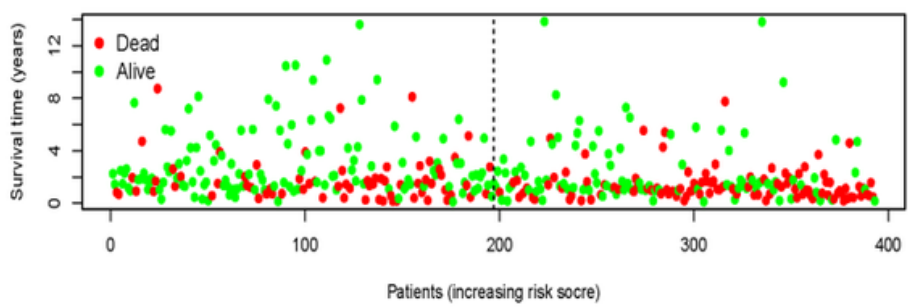

B

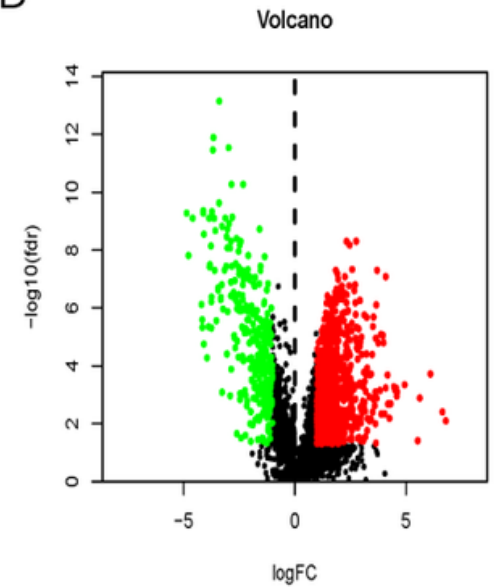

C

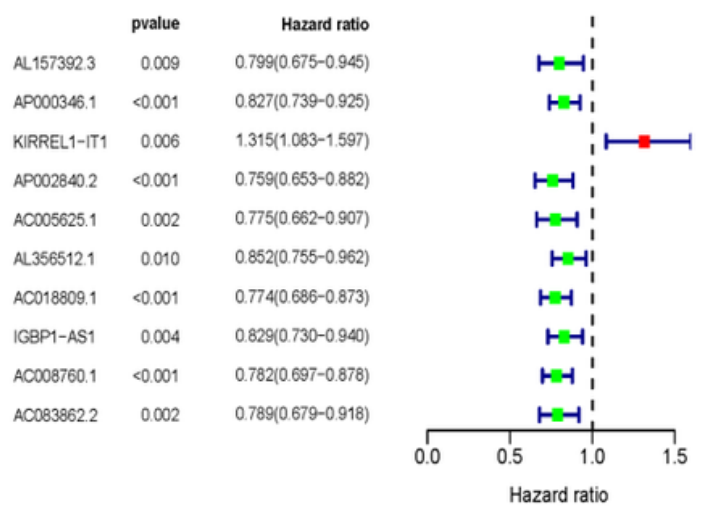

Risk — High risk — Low risk

$\mathrm{F}$

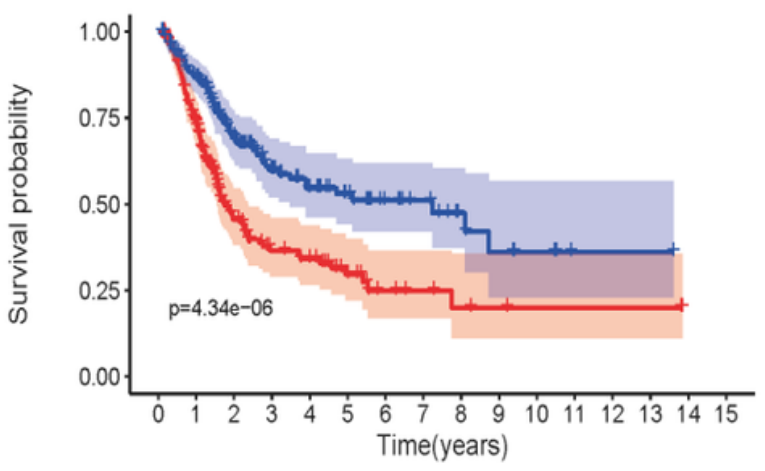

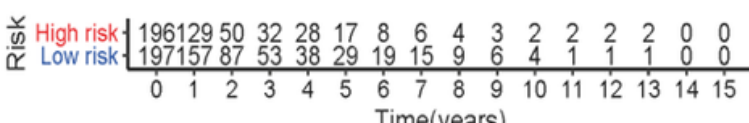
Time(years)

Figure 1

\section{Differentially expressed survival-related ARLNRs in BUC samples and the construction of ARRSM}

Heatmap (A) and volcano plot (B) illustrated the differentially expressed IncRNAs of tumor tissues and adjacent tissues of BUC patients in TCGA database. The red parts represent upregulated IncRNAs, and the blue parts represent downregulated IncRNAs in the heatmap. In the volcano plot, the green dots represent downregulated IncRNAs, the red dots represent the upregulated IncRNAs, and the black dots represent IncRNAs without differential expression. ( $\log 2|\mathrm{FC}|>1$ and $\mathrm{P}<0.05$ ). The hazard ratios of sARLNRs (sARLNRs: AL157392.3, AP000346.1, KIRREL1-IT1, AP002840.2, AC005625.1, AL356512.1, AC018809.1, IGBP1-AS1, AC008760.1 and AC083862.2) 
were demonstrated in the forest plot (C). The green parts represent protective sARLNRs, while the red parts represent deleterious SARLNRs. The risk-score distribution of the high-risk group and low-risk group (D). Survival status of patients in the high-risk group and low-risk group (E). Kaplan-Meier survival curve of the high-risk group and low-risk group $(F)$.

Figure 2

A
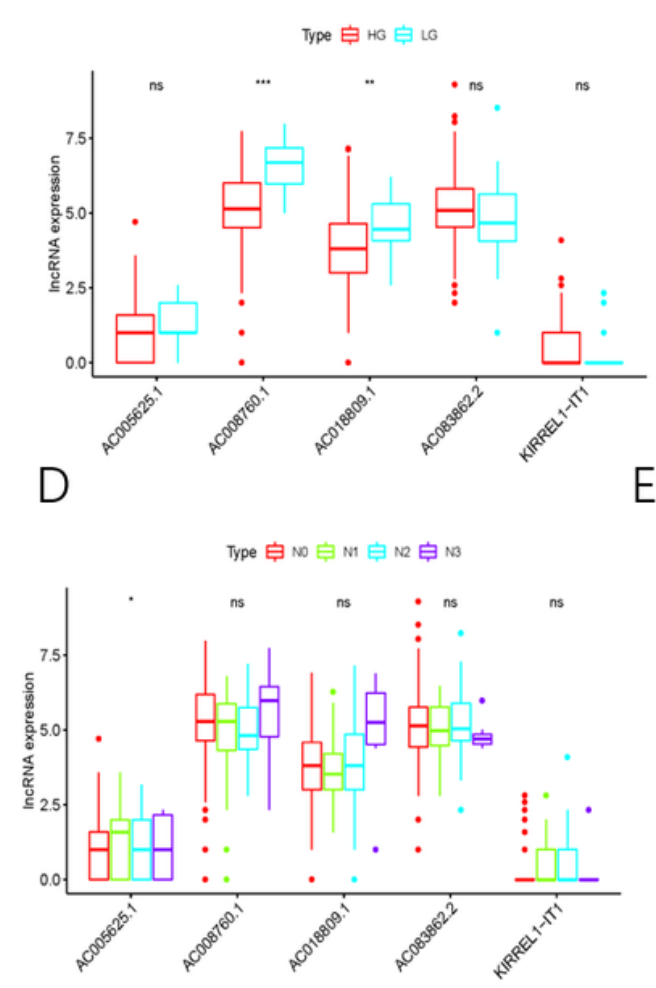

B

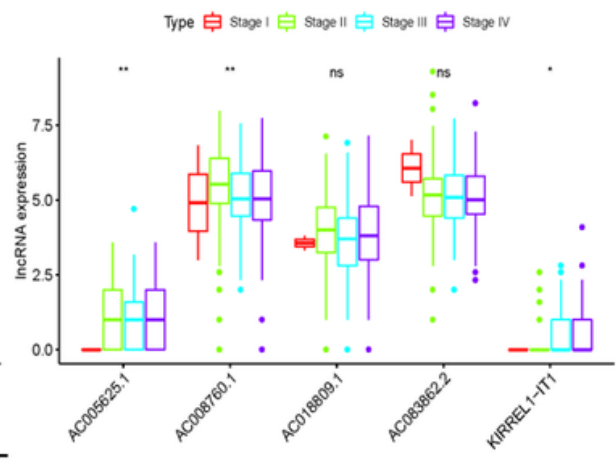

$\mathrm{E}$

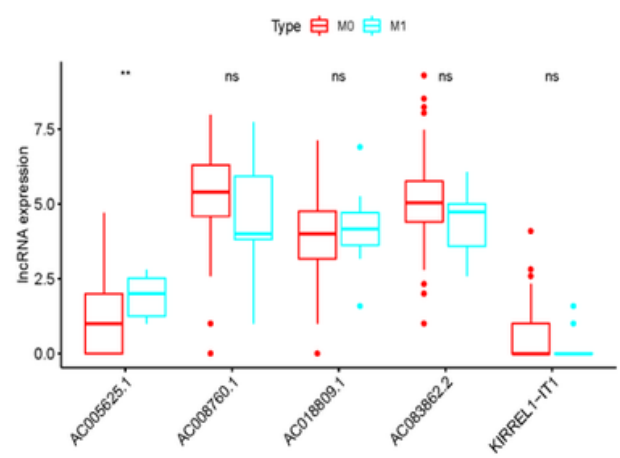

C

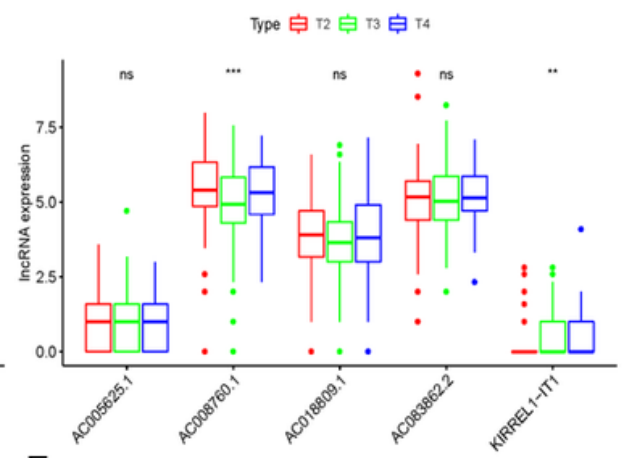

$\mathrm{F}$

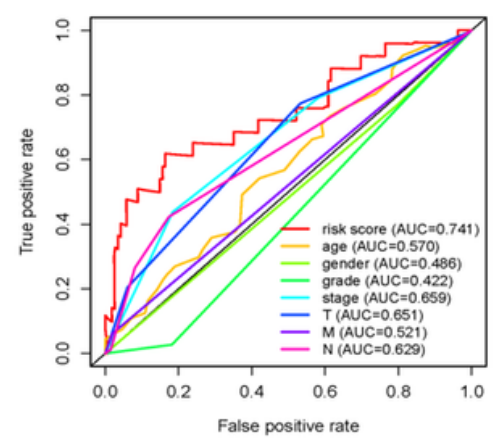

Figure 2

\section{The relationship of ARRSM and clinicopathologic features and the ROC curve of ARRSM}

AC018809.1 and AC008760.1 expressed more in patients with the advanced stage (A). The expression levels of AC005625.1 and AC008760.1 were enhanced in the early stage but KIRREL1-IT1 increased (B). KIRREL1-IT1 expressed more in the advanced T-stage while AC008760.1 dropped (C). The expression levels of AC005625.1 decreased in the advanced $N$-stage but increased in advanced $M$-stage ( $D$ and $E$ ). ( ${ }^{\star * *}: P<0.001, * *$ : $P<0.01, *$ : $P<0.05$, ns: $P>0.05$ ) The areas under curves (AUCs) were evaluated by ROC curves. The AUCs' values of risk score, age, gender, grade, stage, T-stage, M-stage and N-stage were $0.741,0.57,0.486,0.422,0.659,0.651,0.521$ and 0.629 respectively $(F)$. 
Figure 3

A

Points

KIRREL1-IT1

AC005625.1

AC018809.1

AC008760.1

AC083862.2

Total Points

1-year survival

3-year survival

5-year survival

C

Enrichment plot: KEGG_VEGF_SIGNALING_PATHWAY

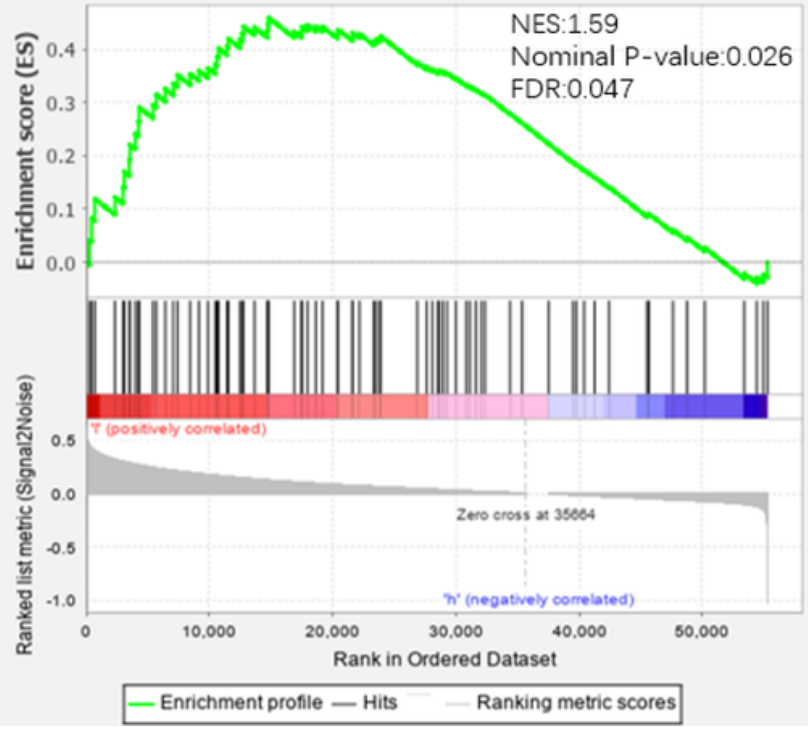

C

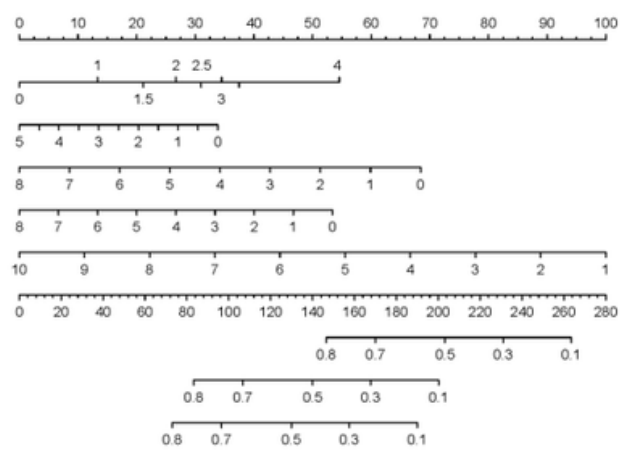

$\mathrm{B}$

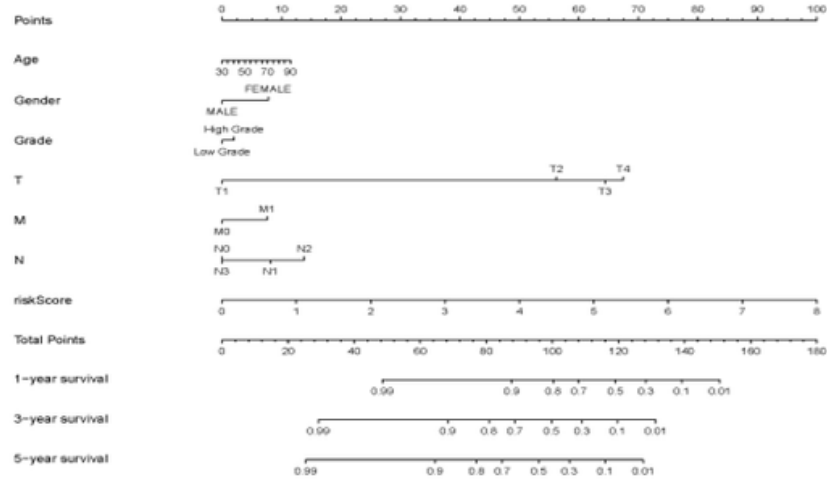


Figure 4

A

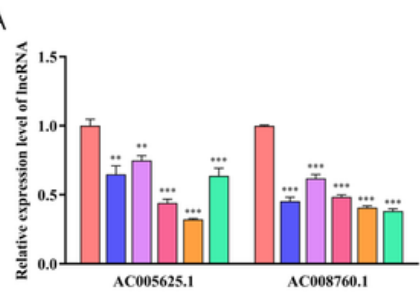

C

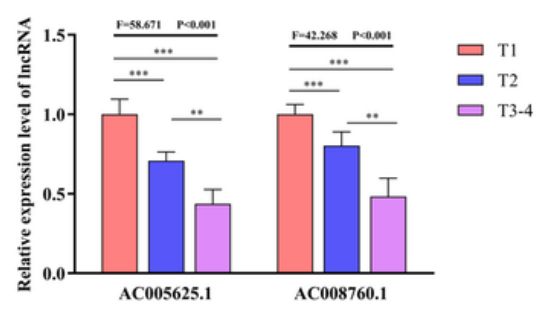

$\mathrm{E}$

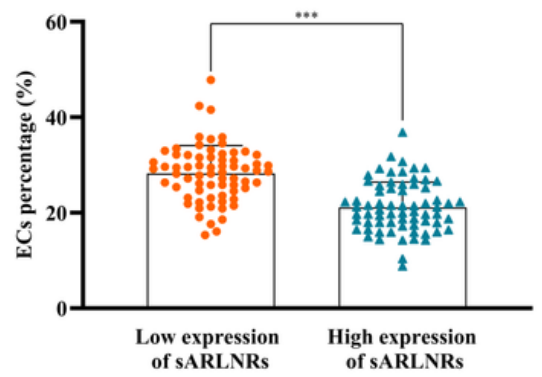

G
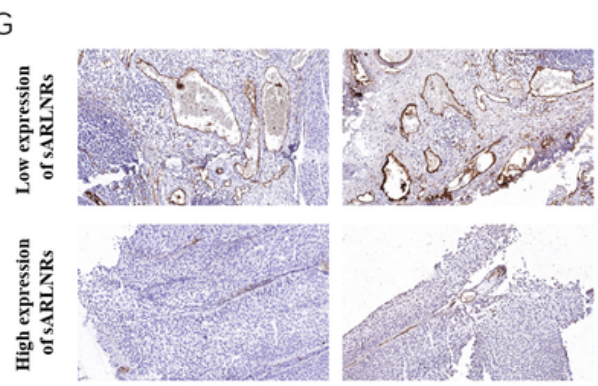

B

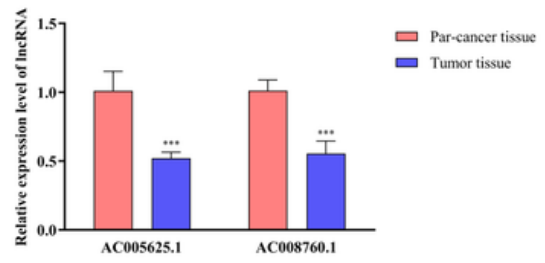

D
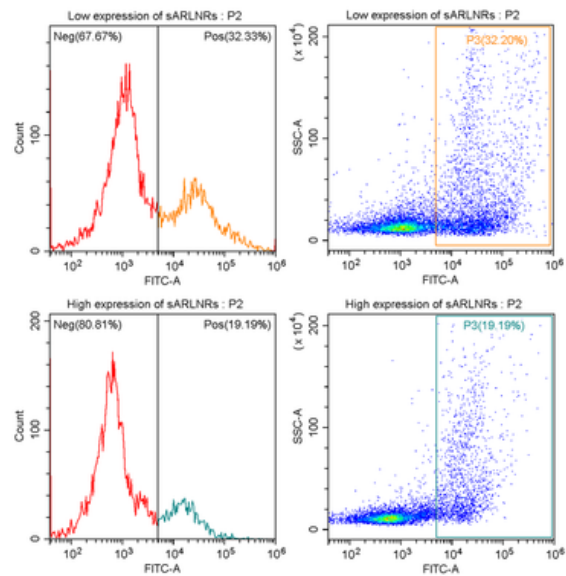

$\mathrm{F}$

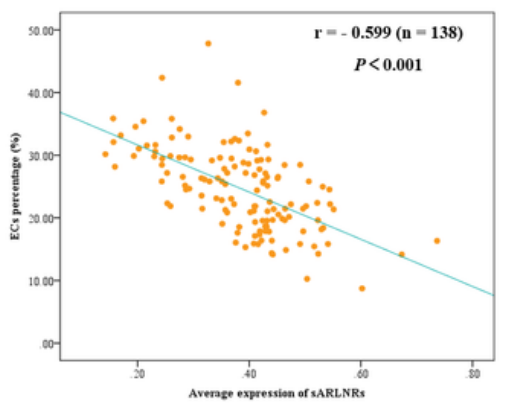

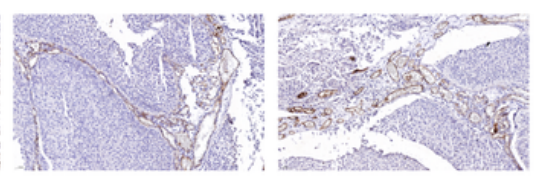
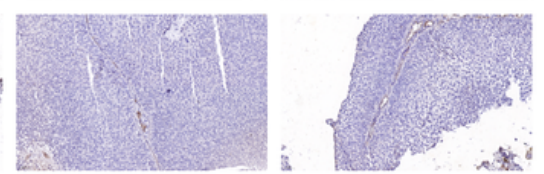

Figure 4

The expression levels of AC005625.1 and AC008760.1 in cell lines and clinical samples.

The qPCR results of the expression levels of AC005625.1 and AC008760.1 in BUC cell lines (T24, 5637, UM-UC-3, 5637, J82 and TCC-SUP) and urothelial cells (SV-HUC-1) $(n=3)(A)$, BUC tumour tissues and adjacent tissues $(n=$ 138) (B and C). The percentage of ECs in BUC tumour tissues with low (top) and high (down) average expression of AC005625.1 and AC008760.1 (D). The quantitative analysis of ECs percentage in BUC tumour tissues $(n=138)(E)$. The Pearson correlation analysis of ECs percentage and average expression of AC005625.1 and AC008760.1 $(n=$ 138 , Pearson's correlation coefficient $=-0.599, P \otimes 0.001)(F)$. IHC was used to determine the EC (identified by CD31 
positivity) percentage in tumour tissues of 138 BUC patients (Scale bar: $50 \mu \mathrm{m})\left({ }^{\star \star *} P<0.001,{ }^{\star \star} P<0.01\right.$ and ${ }^{\star} P<0.05$ represent significant differences between the two groups or compared to Control group).

Figure 5

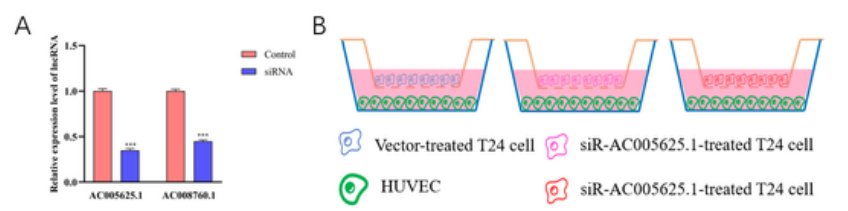

C

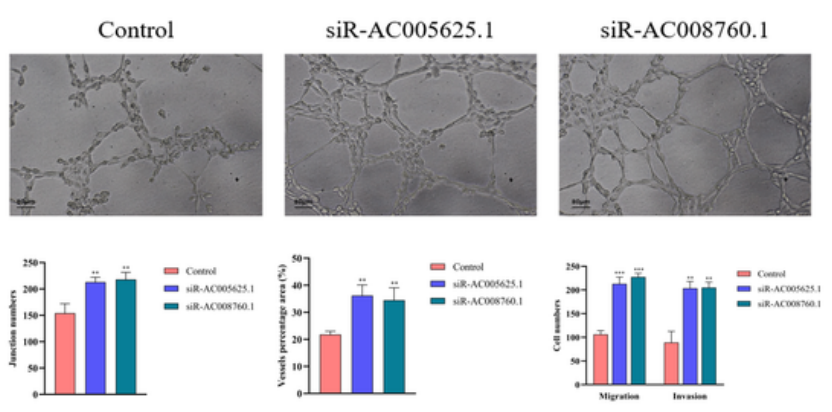

D
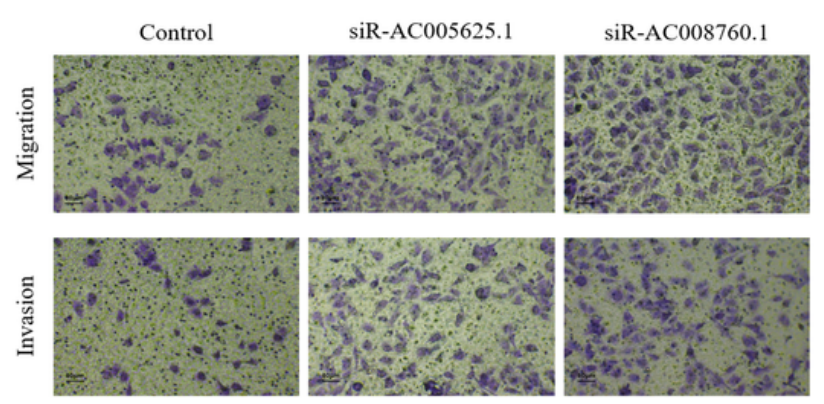

E

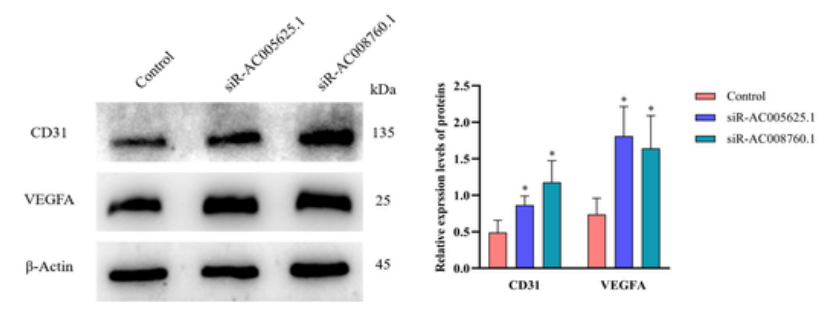

F

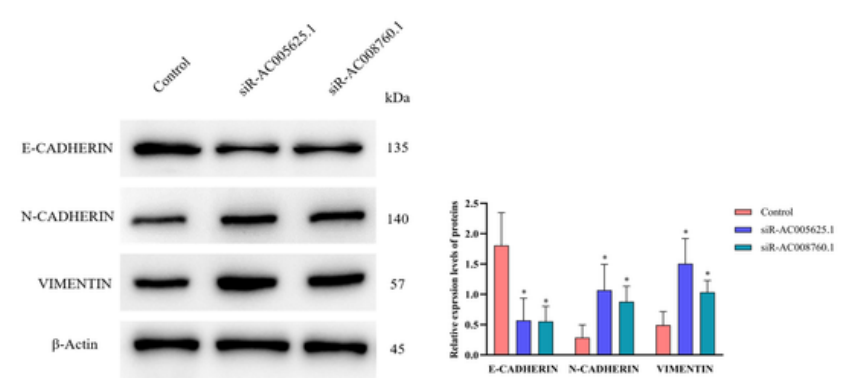

Figure 5

The effects of AC005625.1 and AC008760.1 on tube formation, invasion and migration.

SiR-AC005625.1 and siR-AC008760.1 significantly attenuated the expression of AC005625.1 and AC008760.1 in T24 cells, respectively $(n=3)(A)$. Co-culture models of HUVECs and different T24 cells (B). Knockdown of AC005625.1 and AC008760.1 in T24 cells elevated the tube formation ability of co-cultured HUVECs (top) and quantitative analysis (down) $(\mathrm{n}=3$, Scale bar: $80 \mu \mathrm{m})(\mathrm{C})$. The migration and invasion abilities of HUVEC cells (down) and quantitative analysis (top) $(n=3$, Scale bar: $80 \mu \mathrm{m})$ (D). CD31, VEGF-A, VIMENTIN and N-CADHERIN expressed more 
in HUVECs co-cultured with siR-AC005625.1 and siR-AC008760.1-treated T24 cells, but E-CADHERIN changed reversely (left) and quantitative analysis (right) $(\mathrm{n}=3)(\mathrm{E}$ and $\mathrm{F})$. ( ${ }^{\star \star *} \mathrm{P}<0.001,{ }^{\star} * \mathrm{P}<0.01$ and ${ }^{*} \mathrm{P}<0.05$ represent significant differences compared to Control group).

\section{Supplementary Files}

This is a list of supplementary files associated with this preprint. Click to download.

- FigureS1.tif

- SupplementaryFigureLegend.docx 